EPJ Web of Conferences 32, 02008 (2012)

DOI: $10.1051 /$ epjconf/20123202008

(C) Owned by the authors, published by EDP Sciences, 2012

\title{
A new mechanism for sawtooth period control
}

\author{
M. Lauret ${ }^{1,3, a}$, F. Felici ${ }^{2,3}$, G. Witvoet ${ }^{1,3}$, T. Goodman ${ }^{2}$, G. Vandersteen ${ }^{4}$, E. Westerhof ${ }^{1}$, M.
}

Steinbuch $^{3}$, O. Sauter ${ }^{2}$, M. de Baar ${ }^{1,3}$, and the TCV team ${ }^{2}$

1 FOM Institute DIFFER (Dutch Institute for Fundamental Energy Research) PO Box 1207, 3430 BE Nieuwegein, the Netherlands

2 Ecole Polytechnique Fédérale de Lausanne (EPFL), Centre de Recherches en Physique des Plasmas, Association EURATOM-Confédération Suisse, CH-1015 Lausanne, Switzerland

3 Eindhoven University of Technology (TU/e) PO Box 513, 5600 MB Eindhoven, the Netherlands

4 Vrije Universiteit Brussel, dept. ELEC, Pleinlaan 2, 1050 Brussels, Belgium

\begin{abstract}
Experiments and simulations show that the sawtooth period can be influenced and precisely tuned by modulated ECCD power.
\end{abstract}

\section{Introduction}

In past experiments, the sawtooth period has been controlled by feedback with a variable electron cyclotron current drive (ECCD) deposition location, see e.g.[1]. An alternative is to use open loop control periodically modulating the ECCD power. Simulations [2] predict that for a range of modulation periods and duty cycles (percentage of power on during modulation) the sawtooth period becomes the same as the power modulation period (i.e. sawtooth locking).

Experiments on TCV [3] show indeed convincing evidence of sawtooth locking. In these experiments, the gyrotron power was modulated with periods that were larger than the natural sawtooth period in that operating point. Depending on the duty cycle, locking appeared for certain combinations of the modulation period and duty cycle. The combinations for which locking occurs form a sizeable space in the duty cycle-modulation period graph, the so-called locking range. During a final experiment the modulation period and duty cycle were jointly varied to stay within this locking range and the sawtooth period stayed locked to the modulation period. This open loop control, based on the nonlinear phenomenon of period locking, responds rapidly, often within a few periods, and obviates the slower closed loop sawtooth period control inherent in moving launcher mirrors. It is likely that locking with the input occurs for other periodic processes in the plamsa and that similar methods can be used to control these processes.

This work was supported in part by the Swiss National Science Foundation, NWO, ITER-NL, the European Communities under the contract of the association Euratom/FOM, the Vrije Universiteit Brussel, dept. ELEC, Fund for Scientific Research (FWO-Vlaanderen), the Flemish Government (Methusalem 1), and the Belgian Federal Government.

\section{References}

1. M. Lennholm, L.-G. Eriksson, F. Turco, F. Bouquey, C. Darbos, R. Dumont, G. Giruzzi, M. Jung, R. Lambert, R. Magne, D. Molina, P. Moreau, F. Rimini, J-L. Segui, S. Song, and E. Traisnel Demonstration of Effective Control of Fast-Ion-Stabilized Sawteeth by Electron-Cyclotron Current Drive. Phys. Rev. Lett. 102, 115004 (2009).

\footnotetext{
a e-mail: m. lauret@tue.nl
} 
2. G. Witvoet, M. Lauret, M.R. de Baar, E. Westerhof, M. Steinbuch. Numerical demonstration of injection locking of the sawtooth period by means of modulated EC current drive. Nucl. Fusion $\mathbf{5 1}$ (2011).

3. M. Lauret, F. Felici, G. Witvoet, T.P. Goodman, G. Vandersteen, O. Sauter, M.R. de Baar and the TCV team. Demonstration of sawtooth period locking with power modulation in TCV plasmas. Nucl. Fusion. 52 (2012). 